\title{
EFEITO DE DIFERENTES USOS DO SOLO NA EROSÃO HÍDRICA EM REGIÃO SEMIÁRIDA
}

José Raliuson Inácio Silva ${ }^{1}$, Eduardo Soares de Souza ${ }^{2}$, Rodolfo Souza ${ }^{3}$, Eduardo Silva dos Santos ${ }^{4}$ \& Antonio Celso Dantas Antonino $^{5}$

1 - Engenheiro agrônomo, mestrando em Produção Vegetal, UFRPE/UAST, raliuson.agro@gmail.com

2 - Engenheiro agrônomo, professor associado I, UFRPE/UAST, eduardossouza@ufrpe.br

3 - Engenheiro agrônomo, pós-doutor ONDACBC,UFPE/DEN, rodolfo.marcondes@ufpe.br

4 - Engenheiro agrícola, professor adjunto, UFMA/CCAA, eduardosilva.doctor@gmail.com

5 - Engenheiro civil, professor titular, UFPE/DEN, acdantonino@gmail.com

\section{Palavras-chaves:}

Perda de solo

Caatinga

Escoamento superficial

Pastagem degradada

\section{RESUMO}

Este trabalho teve como objetivo estudar os efeitos do uso diferente da terra na erosão da hídrica sob chuva simulada na região semiárida do Brasil. A pesquisa foi realizada em áreas de caatinga preservada, caatinga sob regeneração, terra arada e pastagem degradada, no município de Serra Talhada, estado de Pernambuco, Brasil. Foi utilizada um simulador de chuvas com intensidade de $100 \mathrm{~mm} \mathrm{~h}^{-1}$ em parcelas com 2,0 $\mathrm{m}^{2}$, com quatro repetições em cada uso da terra, onde foram avaliadas as características hidráulicas do escoamento, taxa de infiltração e perdas de solo e água. O tipo de uso do solo tem efeitos significativos na taxa de infiltração, escoamento e perda de solo. Como esperado, a caatinga preservada obteve a maior taxa de infiltração e as menores perdas de água e solo. A aração não mostrou ser uma boa prática de manejo do solo, uma vez que não reduziu significativamente o escoamento e aumentou a perda de solo cerca de 7,66 vezes mais do que a pastagem degradada. Embora a caatinga regenerada tenha demonstrado baixa capacidade de infiltração e alto escoamento, adquiriu características que diminuem a perda de solo.

\section{Keywords:}

Soil loss

Caatinga

Surface runoff

Degraded pasture

\section{LAND USE EFFECTS ON WATER EROSION IN THE SEMI-ARID REGION OF BRAZIL}

\section{ABSTRACT}

This work aimed to study the effects of different land use on water erosion under the simulated rainfall in the semi-arid region of Brazil. The research was conducted in areas of preserved Caatinga, under-regeneration Caatinga, plowed soil, and degraded grassland in the municipally of Serra Talhada, in State of Pernambuco, Brazil. We used a rainfall simulator with rain intensity of $100 \mathrm{~mm} \mathrm{~h}^{-1}$ in plots measuring $2.0 \mathrm{~m}^{2}$, with four replications per land use, where hydraulic features were assessed as to runoff, infiltration rate, and the soil and water loss. Different soil types have significant effects on the infiltration rate, runoff and soil loss. As we expected, the preserved Caatinga showed the highest infiltration rate and the lowest water and soil losses. Plowing proved not to be a good practice of soil management given it did not reduce runoff significantly and increased soil loss about 7.66 times more than the degraded grassland. Although the regenerated Caatinga showed a low capacity of infiltration and high runoff, it has characteristics that decrease soil loss. 


\section{INTRODUÇÃO}

A erosão hídrica é um dos principais problemas ambientais, pois, além de causar assoreamento dos corpos hídricos, provoca danos irreversíveis nos locais de origem, diminuindo a fertilidade do solo, por remover a camada mais superficial, redução da camada arável e da diversidade e atividade microbiológica do solo, com consequente redução do potencial produtivo do solo, acarretando em maior custo de produção para manutenção da produtividade. No Brasil, vários trabalhos têm encontrado consideráveis valores de perda de solo, sendo mais grave em solos agricultáveis mal manejados (MARIOTI et al., 2013; LIMA et al., 2015; CANTALICE et al., 2016).

O processo de erosão hídrica começa a partir do impacto das gotas de chuva sobre a superfície do solo, sendo que, após a desagregação, o escoamento superficial carrega as partículas de solo soltas e a depositam em outros locais. A vegetação natural proporciona uma barreira física ao escoamento, resultando em redução da velocidade do escoamento e contribuindo, assim, na redução do transporte de sedimentos (RODRIGUES et al., 2013). Contudo, na condução de atividades agropecuárias, muitas vezes o solo fica sem vegetação ao passar por um processo de degradação, ocasionando aumento do escoamento superficial.

Os impactos negativos da erosão hídrica são mais graves em regiões semiáridas, por possuírem solos rasos atrelados à constante remoção da sua vegetação nativa (caatinga) para implantação principalmente de pastagens (FREITAS et al., 2008; AGUIAR et al., 2006), sendo que essas áreas de caatinga têm importantes funções ecológicas como sumidouro de carbono (SILVA et al., 2017a). Apesar da maior parte da precipitação pluvial na região semiárida do Brasil tender a ser convertida em evapotranspiração (SILVA et al., 2017b), a sua elevada sazonalidade proporciona valores extremos de chuva (SANTOS et al., 2017), com consequente aumento do escoamento superficial.

A remoção da vegetação nativa, associada à elevada sazonalidade das chuvas, promove condições favoráveis para aumento do escoamento superficial. Leite et al. (2017), que estudaram escoamento superficial em diferentes coberturas de solo na região semiárida do Nordeste brasileiro, encontraram que áreas com caatinga tendem a ter um menor escoamento superficial de água. Já Souza et al. (2015) verificaram que $26 \%$ da precipitação pluvial é perdida por escoamento superficial, em pastagem na região semiárida brasileira. No entanto, ainda são poucos os estudos que avaliam o impacto da substituição da caatinga por atividades agropecuárias, sob o escoamento e erosão do solo. A principal causa para escassez de informações está relacionada ao trabalho árduo e demora para aquisição desses dados sob condições naturais.

Assim, a utilização de simuladores de chuvas tem surgido como uma alternativa para uma rápida aquisição de dados sobre escoamento superficial. Além disso, esses equipamentos apresentam a grande vantagem de permitir estudar simultaneamente o escoamento superficial, a infiltração da água no solo e a perda de solo. Diante desse contexto, o presente trabalho teve como objetivo estudar a erosão hídrica em quatro diferentes usos de solo no semiárido pernambucano: uma pastagem degradada, uma área de terra arada e duas áreas de caatinga (com diferentes estágios sucessionais) sob condições de chuva simulada.

\section{MATERIAL E MÉTODOS}

O trabalho foi conduzido nas fazendas Buenos Aires e Alagoinha, ambas situadas no município de Serra Talhada-PE. O clima da região é do tipo BShw', semiárido, caracterizando-se por ser quente e seco, com as chuvas de verão ocorrendo entre os meses de dezembro a maio, sendo sua precipitação média anual de aproximadamente $642 \mathrm{~mm}$ (SILVA et al., 2015).

O estudo foi conduzido em quatro usos de solo comuns no semiárido, sendo uma pastagem degradada (PD), onde inicialmente foi implantado o capim corrente (Urochloa mosambicensis) e atualmente tem predomínio de uma planta daninha popularmente conhecida como malva (Waltheria indica L.); uma área de terra arada (TA), que estava em processo de regeneração natural a três anos; uma caatinga que passou por um processo de regeneração natural (CR) por 30 anos, com predomínio de angico (Anadenanthera colubrina (Vell.) Brenan); e uma área de caatinga preservada 
(CP), com cobertura vegetal não muito densa, mas predominantemente arbórea. Todas as áreas eram utilizadas para pastejo animal e possuem as características físicas e texturais descritas na Tabela 1.

O simulador de chuvas utilizado foi um sistema hidráulico, composto por três emissores (um com leque de $360^{\circ}$, localizado no centro, e dois de $180^{\circ}$, nas extremidades) acoplados a uma motobomba de $360 \mathrm{~W}(1 / 2 \mathrm{CV})$, que fornecia água sob uma pressão constante de $20 \mathrm{KPa}$. Durante a simulação, os emissores ficaram a uma altura de $1,85 \mathrm{~m}$ da superfície do solo. Em ambiente controlado e sem interferências de ventos, a intensidade de chuvas foi igual a $100 \mathrm{~mm} \mathrm{~h}^{-1}$, com uma uniformidade de distribuição de água de $77 \%$. Essa uniformidade é superior à encontrada por Abrantes et al. (2015), que, estudando o escoamento superficial por chuva simulada, obtiveram uniformidade variando de 37,3 a $47,1 \%$.

Em cada área, foram escolhidas, ao acaso, quatro parcelas experimentais delimitadas por chapas galvanizadas de $2 \mathrm{~m}$ de comprimento, $1 \mathrm{~m}$ de largura e $0,12 \mathrm{~m}$ de altura, cravadas no solo a uma profundidade de $5 \mathrm{~cm}$, com a maior dimensão no sentido do declive. Na parte inferior das parcelas, uma calha coletora permitiu medir a vazão do escoamento superficial e coletar os sedimentos. Ao lado das calhas de escoamento foram distribuídos 10 pluviômetros para a aferição da intensidade média das chuvas simuladas.

Antes do início de cada simulação, em cada parcela experimental, foram determinadas a declividade, a porcentagem de cobertura vegetal e a umidade inicial do solo obtidas a partir de amostras de solo coletadas de 0 a $10 \mathrm{~cm}$. A porcentagem de cobertura vegetal foi definida com uma moldura de $50 \times 50 \mathrm{~cm}$, colocada em uma porção representativa da parcela experimental, da qual foram retiradas fotografias. As imagens resultantes foram processadas pelo programa ImageJ (RASBAND, 2012), que possibilitou determinar as suas respectivas porcentagens de cobertura.

Quando a água começou a fluir na calha da coleta, mediu-se a vazão de escoamento superficial a cada 5 minutos, sendo que, para essa determinação, foram coletadas amostras durante 20 segundos em recipientes de um litro; após a coleta, o volume escoado era aferido. O coeficiente de escoamento superficial (C) foi obtido pela relação entre a taxa de escoamento superficial $\left(\mathrm{mm} \mathrm{h}^{-1}\right)$ e a intensidade de precipitação $\left(\mathrm{mm} \mathrm{h}^{-1}\right)$. A lâmina de água infiltrada foi calculada pela diferença entre a lâmina de água aplicada e a lâmina de escoamento superficial, para cada intervalo de coleta. Os valores de taxa de infiltração foram obtidos pela relação entre lâmina infiltrada e o tempo de simulação da chuva.

A concentração de sedimentos e as taxas de perdas de solo e de desagregação foram obtidas por pesagem do material coletado nos potes plásticos. $\mathrm{O}$ volume de água e sedimentos coletados passaram 72 horas em repouso para retirada do sobrenadante e, em seguida, os mesmos foram encaminhados para estufa a $40^{\circ} \mathrm{C}$. Transcorrido o tempo necessário para evaporação de toda a água, os recipientes foram pesados novamente, desta vez já com o solo seco. A relação entre a massa de solo seco e o volume da mistura de água e sedimentos, expressa a concentração de sedimentos em $\mathrm{kg} \mathrm{L}^{-1}$.

A taxa de desagregação do solo, $\mathrm{D}_{\mathrm{e}}\left(\mathrm{kg} \mathrm{m}^{-2} \mathrm{~s}^{-1}\right)$, foi determinada conforme a expressão:

$\mathrm{D}_{\mathrm{e}}=\frac{\mathrm{Mss}}{\mathrm{A} \mathrm{D}_{\mathrm{c}}}$

sendo, Mss a massa do solo seco desagregado $(\mathrm{kg})$, $\mathrm{A}$ a área da parcela $\left(\mathrm{m}^{2}\right)$ e $\mathrm{D}_{\mathrm{c}}$ a duração da coleta $(\mathrm{s})$.

Tabela 1. Características físicas e texturais das áreas de caatinga preservada $(\mathrm{CP})$, caatinga regenerada (CR), terra arada (TA) e pastagem degrada (PD) na camada de $0-10 \mathrm{~cm}$

\begin{tabular}{cccccl}
\hline Áreas & Argila & Silte & Areia & Ds & Classe textural \\
\hline & $\mathrm{g} \mathrm{kg}^{-1}$ & $\mathrm{~g} \mathrm{~kg}^{-1}$ & $\mathrm{~g} \mathrm{~kg}^{-1}$ & $\mathrm{~g} \mathrm{~cm}^{-3}$ & \\
CP & 111,04 & 172,44 & 716,52 & 1,39 & Franco arenoso \\
CR & 109,43 & 206,86 & 683,67 & 1,39 & Franco arenoso \\
TA & 161,42 & 202,87 & 635,70 & 1,40 & Franco arenoso \\
PD & 167,30 & 166,31 & 666,38 & 1,44 & Franco arenoso \\
\hline
\end{tabular}


A perda de solo, $\operatorname{Ps}\left(\mathrm{kg} \mathrm{m}^{-2}\right)$, foi estimada pela seguinte equação:

$$
P_{s}=\frac{\left(\mathrm{QC}_{\mathrm{s}} \mathrm{t}\right)}{\mathrm{A}}
$$

sendo, Q a vazão de escoamento $\left(\mathrm{L} \mathrm{s}^{-1}\right)$, Cs a concentração de sedimentos $\left(\mathrm{kg} \mathrm{L}^{-1}\right)$ e t o intervalo de tempo entre as coletas (300 s).

Quando as intensidades de precipitação observadas foram abaixo da planejada $(100 \mathrm{~mm}$ $\left.\mathrm{h}^{-1}\right)$, a taxa de desagregação do solo $\left(\mathrm{D}_{\mathrm{e}}\right)$ e a de perda de solo $\left(\mathrm{P}_{\mathrm{s}}\right)$ foram normalizadas (CASSOL et al., 2004):

$D_{\mathrm{en}}=D_{e}\left(\frac{I_{p}}{I_{o}}\right)^{2}$

$P_{\mathrm{sn}}=\operatorname{Ps}\left(\frac{I_{p}}{I_{o}}\right)^{2}$

sendo, $\mathrm{D}_{\text {en }}\left(\mathrm{kg} \mathrm{m}^{-2} \mathrm{~s}^{-1}\right)$ a taxa de desagregação do solo normalizada, $\mathrm{P}_{\mathrm{sn}}\left(\mathrm{kg} \mathrm{m}^{-2}\right)$ a perda de solo normalizada, $I_{p}\left(\mathrm{~mm} \mathrm{~h}^{-1}\right)$ a intensidade de precipitação planejada e $\mathrm{I}_{\mathrm{o}}\left(\mathrm{mm} \mathrm{h}^{-1}\right)$ a intensidade de precipitação observada.

De posse dos dados de escoamento e de perda de solo, pôde-se determinar a eficiência na redução de escoamento (ERE) e de sedimentos (ERS) (ZHAO et al., 2014):

$\mathrm{ERE}=\left(\frac{E_{0}-E_{i}}{E_{0}}\right) 100$

$\mathrm{ERS}=\left(\frac{S_{0}-S_{i}}{S_{0}}\right) 100$

sendo, $\mathrm{E}_{0}(\mathrm{~mm})$ o escoamento da área com maior volume escoado, $\mathrm{E}_{\mathrm{i}}(\mathrm{mm})$ o escoamento da área que se deseja obter a ERE, $\mathrm{S}_{0}(\mathrm{~kg})$ a perda de solo da área com maior produção de sedimentos e $\mathrm{S}_{\mathrm{i}}(\mathrm{kg})$ a perda de solo da área que se deseja obter a ERS.

Durante as simulações de chuva também foi possível determinar as características hidráulicas do escoamento. A velocidade superficial do escoamento $\left(\mathrm{m} \mathrm{s}^{-1}\right)$ foi determinada analisandose o tempo necessário para que o corante (azul de metileno) percorresse uma distância de $40 \mathrm{~cm}$ na parcela experimental. Essa etapa foi realizada em intervalos de 5 minutos, após a formação e o deslocamento da lâmina de escoamento. Os valores de velocidade superficial do escoamento foram, em seguida, multiplicados por um fator de correção $(\alpha=2 / 3)$, para obtenção da velocidade média do escoamento, $\mathrm{V}_{\mathrm{m}}\left(\mathrm{m} \mathrm{s}^{-1}\right)$ (BEZERRA; CANTALICE, 2006).

A altura da lâmina do escoamento $\mathrm{h}$ foi determinada pela equação (1), derivada por Woolhiser e Liggett (1967) e Singh (1983) para fluxo em um plano sob chuva de duração finita:

$$
\mathrm{h}=\frac{\mathrm{q}}{\mathrm{Vm}}
$$

sendo $\mathrm{h}$ a altura da lâmina de escoamento (m), q a descarga líquida por unidade de largura $\left(\mathrm{m}^{2} \mathrm{~s}^{-1}\right)$ e $\mathrm{V}_{\mathrm{m}}$ a velocidade média do escoamento $\left(\mathrm{m} \mathrm{s}^{-1}\right)$. A descarga líquida (q) foi obtida pela divisão da taxa de escoamento total pela largura da parcela.

$\mathrm{O}$ número de Reynolds (Re) foi estabelecido pela seguinte relação (SIMONS; SENTURK, 1992):

$\mathrm{R}_{\mathrm{e}}=\frac{\mathrm{V}_{\mathrm{m}} \mathrm{h}}{\mathrm{v}}$

sendo, $\mathrm{v}$ viscosidade cinemática da água $\left(\mathrm{m}^{2} \mathrm{~s}^{-1}\right)$ definida por (JULIEN, 1995):

$$
v=\left[1,14-0,031(\mathrm{~T}-15)+(\mathrm{T}-15)^{2}\right] 10^{-6}
$$

sendo, $\mathrm{T}$ a temperatura da água em ${ }^{\circ} \mathrm{C}$ aferida durante cada simulação, por meio de um termômetro colocado em um dos pluviômetros posicionado ao lado da parcela experimental.

O número de Froude ( $\mathrm{Fr}$ ) foi definido conforme Simons e Senturk (1992):

$$
F_{r}=\frac{\mathrm{Vm}}{\sqrt{\mathrm{gh}}}
$$

sendo, $\mathrm{g}$ a aceleração da gravidade $\left(\mathrm{m} \mathrm{s}^{-2}\right)$.

Os números de Reynolds e Froude, auxiliaram na definição dos regimes de escoamento gerados a partir das chuvas simuladas: i) quando $\operatorname{Re}<500 \mathrm{e}$ $\mathrm{Fr}<1$, o regime de escoamento é laminar lento; ii) enquanto $\operatorname{Re}<500$ e $\mathrm{Fr}>1$, o regime é considerado laminar rápido (SIMONS; SENTURK, 1992). 
Os resultados foram submetidos ao teste de normalidade de Kolmogorov-Smirnov, à análise de variância (teste F a 5 \%) e ao teste de Tukey (5\%) para comparação das médias. Para todas as análises estatísticas e confecção dos gráficos, utilizou-se o software R (R CORE TEAM, 2015).

\section{RESULTADOS E DISCUSSÃO}

Na Tabela 2 estão dispostas as características das parcelas experimentais e da chuva simulada. Não houve diferenças para a declividade do solo e para a porcentagem de cobertura do solo. Essa padronização é importante, visto que diferentes declividades e porcentagens de cobertura influenciam de forma significativa na perda de solo, infiltração e características hidráulicas do escoamento (CANTALICE et al., 2016). A umidade inicial, fator que influencia principalmente o tempo de início do escoamento, apresentou diferença entre as áreas estudadas, sendo que a caatinga preservada (CP) apresentou o menor valor, porém não diferiu da pastagem degradada (PD).

As intensidades de precipitação foram menores do que a planejada $\left(100 \mathrm{~mm} \mathrm{~h}^{-1}\right)$ e diferiram entre os tratamentos (Tabela 2), sendo esse fato também observado por Casssol et al. (2003, 2004). As menores intensidades de precipitação registradas na pastagem degradada (PD) e na terra arada (TA) se devem à interferência do vento, visto que essas áreas são mais susceptíveis a essa variável, por não possuírem vegetação arbórea capaz de diminuir a velocidade dos ventos. Nas áreas de $\mathrm{CP}$ e $\mathrm{CR}$, a intensidade observada se aproximou da projetada, pois a vegetação arbórea corrobora para menor interferência dos ventos. A lâmina precipitada seguiu o mesmo comportamento da intensidade das chuvas, diferenciando entre as áreas estudadas.
A taxa de infiltração inicial $\left(i_{0}\right)$ foi menor na área de $\mathrm{PD}$, diferindo estatisticamente para as demais áreas (Tabela 3). A aração não se mostrou uma prática eficiente para aumentar a $\mathrm{i}_{0}$, uma vez que não houve diferença entre a PD e a TA. Como já era esperado, a CR e a CP obtiveram os maiores valores de $\mathrm{i}_{0}$. Apesar dos valores de umidade inicial terem sido diferentes entre as áreas de $\mathrm{CP} \mathrm{e}$ de CR (Tabela 2), a i entre elas não foi diferente, demonstrando que a infiltração é um processo dinâmico que pode ser influenciado por outros fatores, além da umidade do solo na fase inicial.

Com relação à taxa de infiltração final $\left(i_{f}\right)$, houve diferença entre as áreas, com a $\mathrm{CP}$ se destacando em relação às demais, superando em 145,111 e $115 \%$ a $i_{f}$ das áreas de PD, TA e CR, respectivamente. Santos et al. (2008), avaliando a erosão hídrica em diferentes coberturas de solo no semiárido, encontraram taxa de infiltração de 54,94 $\mathrm{mm} \mathrm{h}^{-1}$, bem próximo do reportado neste estudo. Esses valores foram menores do que os reportados por Cantalice et al. (2008), que, ao avaliarem o escoamento em área de caatinga, encontraram taxas de infiltração na ordem de $125 \mathrm{~mm} \mathrm{~h}^{-1}$.

Vale salientar que as áreas de CR e CP são utilizadas para pastejo animal seguindo a sazonalidade das chuvas, ou seja, no período chuvoso, enquanto as áreas de PD e TA foram frequentemente pastejadas. De acordo com Parente et al. (2010), o impacto do pisoteio animal causa alterações nas propriedades físico-hídricas do solo, provocando diminuição da taxa de infiltração de água no solo. A CR apresentou $\mathrm{i}_{0}$ próximos da $\mathrm{CP}$, porém esse comportamento não se repetiu para $i_{f}$, demonstrando que, mesmo decorridos 30 anos, a CR não ostenta as características de infiltração de uma CP.

Tabela 2. Características das parcelas experimentais e das chuvas simuladas, em uma pastagem degradada $(\mathrm{PD})$, terra arada (TA), caatinga regenerada (CR) e caatinga preservada (CP)

\begin{tabular}{cccccc}
\hline Tratamentos & Intensidade $\left(\mathrm{mm} \mathrm{h}^{-1}\right)$ & Precipitação $(\mathrm{mm})$ & Declividade (\%) & Cobertura (\%) & Umidade inicial $(\mathrm{g} / \mathrm{g})$ \\
\hline $\mathrm{PD}$ & $74,86 \mathrm{~b}$ & $60,42 \mathrm{~b}$ & $3,92 \mathrm{a}$ & $58,36 \mathrm{a}$ & $0,021 \mathrm{ab}$ \\
$\mathrm{TA}$ & $78,18 \mathrm{~b}$ & $66,07 \mathrm{~b}$ & $3,32 \mathrm{a}$ & $51,43 \mathrm{a}$ & $0,033 \mathrm{a}$ \\
$\mathrm{CR}$ & $94,11 \mathrm{a}$ & $80,24 \mathrm{a}$ & $5,12 \mathrm{a}$ & $50,17 \mathrm{a}$ & $0,038 \mathrm{a}$ \\
$\mathrm{CP}$ & $90,42 \mathrm{a}$ & $81,18 \mathrm{a}$ & $4,09 \mathrm{a}$ & $54,03 \mathrm{a}$ & $0,013 \mathrm{~b}$ \\
\hline
\end{tabular}

Médias seguidas pela mesma letra na coluna não diferem entre si, pelo teste de Tukey a 5\%. 


\section{EFEITO DE DIFERENTES USOS DO SOLO NA EROSÃO HÍDRICA EM REGIÃO SEMIÁRIDA}

Na Tabela 4 são apresentadas as características hidráulicas do escoamento superficial para as quatro áreas. A velocidade média do escoamento $(\mathrm{Vm})$ foi diferente apenas para TA estatisticamente das demais áreas, a Vm foi favorecida na TA pelos pequenos sulcos formados no sentido do declive durante o preparo do solo; como esses sulcos não oferecem resistência ao escoamento, o mesmo tende a ganhar velocidade. As demais áreas não diferem entre si e os valores de $\mathrm{Vm}$ variam de 0,01 a 0,027 $\mathrm{m} \mathrm{s}^{-1}$. Os valores de $\mathrm{Vm}$ das áreas de PD e de CP foram menores do que os achados por Cantalice et al. (2016) e Freitas et al. (2008), que encontraram valores de 0,035 e $0,023 \mathrm{~m} \mathrm{~s}^{-1}$, estudando áreas de pastagem e caatinga, respectivamente. No entanto, essa diferença pode estar relacionada às condições de solo, cobertura e declividade que podem ou não favorecer o escoamento.

Encontraram-se valores de descarga líquida por unidade de largura na ordem de $10^{-5} \mathrm{~m}^{2} \mathrm{~s}^{-1}$, estando de acordo com os detectados por Lima et al. (2015) e Franco et al. (2012). A área de $\mathrm{CP}$ apresentou menor valor $\mathrm{q}$, fato esse também observado por Santos et al. (2009). Essa menor descarga é justificada pela taxa de infiltração na $\mathrm{CP}$ ser superior às demais áreas (Tabela 3 ), conforme mais água vai sendo infiltrada, menos fica disponível para o escoamento e consequentemente para descarga líquida. Com relação à altura do escoamento (h), a TA obteve menor valor $(0,26$ $\mathrm{mm})$, diferenciando-se das demais áreas. Freitas et al. (2008) ressaltam que quanto menor a resistência ao escoamento, menor a altura do mesmo. Como os pequenos sulcos deixados após o preparo do solo não oferecem resistência ao escoamento, essa menor altura encontrada na TA é compreensível.

De posse do número de Reynolds $(\mathrm{Re})$ e de Froude (Fr), possibilitou-se a identificação do regime de escoamento como laminar lento nas áreas de PD, CR e CP, de acordo com a classificação proposta por Simons e Senturk (1992), onde $\operatorname{Re}<500$ e $\mathrm{Fr}<1$, característica de escoamento que indica a ocorrência de erosão entressulcos. Esse tipo de erosão hídrica é considerado o mais danoso, por remover a camada mais superficial, onde estão a maior parte dos nutrientes (LIMA et al., 2015). A exceção ocorreu na TA, que foi caracterizada como laminar rápido, possuindo $\mathrm{Re}$ $<500$ e $\mathrm{Fr}>1$, o aumento no número de $\mathrm{Fr}$ em comparação às demais áreas decorreu devido à ausência de barreiras que oferecessem resistência ao escoamento. Assim como observado neste trabalho, Freitas et al. (2008), estudando a erosão entressulcos sob caatinga e cultivos agrícolas, encontraram o menor número de Re para o solo ocupado com caatinga.

Tabela 3. Taxa de infiltração de água no solo obtida por simulação de chuva, em uma pastagem degradada $(\mathrm{PD})$, terra arada (TA), caatinga regenerada (CR) e caatinga preservada (CP)

\begin{tabular}{ccccc}
\hline Taxas de infiltração $\left(\mathrm{mm} \mathrm{h}^{-1}\right)$ & PD & TA & CR & CP \\
\hline $\mathrm{i}_{0}-$ inicial & $61,5 \mathrm{~b}$ & $68,3 \mathrm{ba}$ & $83,3 \mathrm{a}$ & $82,6 \mathrm{a}$ \\
$\mathrm{i}_{\mathrm{f}}$ - final & $21,8 \mathrm{~b}$ & $25,3 \mathrm{~b}$ & $24,8 \mathrm{~b}$ & $53,6 \mathrm{a}$ \\
\hline
\end{tabular}

Médias seguidas pela mesma letra na linha não diferem entre si pelo teste de Tukey a 5\%.

Tabela 4. Características hidráulicas do escoamento nas áreas pastagem degradada (PD), terra arada (TA), caatinga regenerada $(\mathrm{CR})$ e caatinga preservada $(\mathrm{CP})$

\begin{tabular}{ccccccc}
\hline Áreas & $\mathrm{Vm}\left(\mathrm{m} \mathrm{s}^{-1}\right)$ & $\mathrm{q}\left(\mathrm{m}^{2} \mathrm{~s}^{-1}\right)$ & $\mathrm{h}(\mathrm{mm})$ & $\operatorname{Re}(\mathrm{adm})$. & $\operatorname{Fr}(\mathrm{adm})$. & $v\left(\mathrm{~m}^{2} \mathrm{~S}^{-1}\right)$ \\
\hline PD & $0,010 \mathrm{~b}$ & $1,51 \times 10^{-5} \mathrm{ab}$ & $1,38 \mathrm{a}$ & $17,27 \mathrm{ab}$ & $0,09 \mathrm{~b}$ & $8,30 \times 10^{-7} \mathrm{c}$ \\
$\mathrm{TA}$ & $0,045 \mathrm{a}$ & $1,45 \times 10^{-5} \mathrm{ab}$ & $0,26 \mathrm{~b}$ & $17,45 \mathrm{ab}$ & $1,18 \mathrm{a}$ & $9,20 \times 10^{-7} \mathrm{a}$ \\
$\mathrm{CR}$ & $0,027 \mathrm{~b}$ & $1,85 \times 10^{-5} \mathrm{a}$ & $1,32 \mathrm{a}$ & $18,54 \mathrm{ab}$ & $0,15 \mathrm{~b}$ & $9,00 \times 10^{-7} \mathrm{ab}$ \\
$\mathrm{CP}$ & $0,013 \mathrm{~b}$ & $1,02 \times 10^{-5} \mathrm{~b}$ & $0,98 \mathrm{a}$ & $15,64 \mathrm{~b}$ & $0,12 \mathrm{~b}$ & $8,80 \times 10^{-7} \mathrm{~b}$ \\
\hline
\end{tabular}

Médias seguidas pela mesma letra na coluna não diferem entre si pelo teste de Tukey a 5\%. Velocidade média do escoamento (Vm), descarga por unidade de largura (q), altura do escoamento (h), número de Reynolds (Re), número de Froude (Fr) e viscosidade sinemática (v). 
A viscosidade cinemática da água, parâmetro utilizado para obtenção do número de Re, diferenciou entre as áreas. Estando essas diferenças relacionadas ao fato das simulações terem sido realizadas em horários e dias diferentes em cada área estudada, como a mesma depende exclusivamente da temperatura, que varia ao longo do dia e entre os dias, é aceitável ocorrer essa diferença entre os valores de viscosidade.

$\mathrm{Na}$ Tabela 5, são apresentadas as variáveis relacionadas à taxa de erosão do solo e escoamento superficial. A PD teve o menor tempo de início do escoamento (TI), seguida pela TA, CR e CP, sendo que para essa última a TI foi 2,5 vezes maior em comparação com a PD. A TA não se mostrou um tratamento eficaz para aumento do TI, visto que a mesma não se diferenciou da PD. Panachuki et al. (2011) observaram que a aração só aumentou o tempo de início do escoamento, quando a mesma foi associada a doses de cobertura na superfície do solo. O TI torna-se importante em estudos hidrológicos, pois quanto maior o tempo para início do escoamento, menor será a possibilidade de ocorrência da erosão hídrica.

Em relação ao coeficiente de escoamento superficial (C), a área de CP apresentou menor valor, sendo esse muito próximo do encontrado por Cantalice et al. (2008), que estudaram o escoamento em área de caatinga e encontraram $\mathrm{C}$ igual a 0,42. Esses autores ressaltam que o processo de infiltração de água no solo, nas áreas de caatinga, é favorecido pelo fato de o solo dessas áreas não passar por práticas agrícolas. Os demais tratamentos não diferem entre si, apresentando C entre 0,67 e 0,74 (Tabela 5).

Comparando-se os valores médios de perda de solo, nota-se que a TA apresentou a maior perda de solo, de $144,69 \mathrm{Kg} \mathrm{ha}^{-1}$, fato esse também encontrado por Panachuki et al. (2011). Com a aração ocorre a quebra dos agregados do solo, o que facilita o desprendimento, a suspensão e o carreamento por escoamento superficial. Assim como observado por Lima et al. (2013), a CP obteve a menor perda de solo, de 7,26 kg ha-1. Esta não diferiu da CR, mostrando que uma área anteriormente degradada, após passar por um processo de regeneração natural, pode adquirir características de resistência à perda de solo, semelhantes a uma caatinga preservada. Corroborando nosso trabalho, Santos et al. (2009) constataram perda de solo de $10 \mathrm{~kg} \mathrm{ha}^{-1}$ para área com cobertura natural no semiárido, sendo esse valor próximo ao encontrado neste trabalho.

Seguindo o mesmo comportamento da perda de solo, a taxa de desagregação foi maior na TA e menor na $\mathrm{CP}$ e na $\mathrm{CR}$, ficando a $\mathrm{PD}$ com valor intermediário. A taxa de desagregação da PD foi inferior à identificada por Cantalice et al. (2016), que, ao estudarem a erosão hídrica em pastagem, encontraram taxa de desagregação na ordem de 5,66 $\times 10^{-5} \mathrm{~kg} \mathrm{~m}^{-2} \mathrm{~s}^{-1}$. Esses autores avaliaram a perda de solo em diferentes declividades, sendo o referido valor obtido com a declividade de $15 \%$, bem maior ao da presente pesquisa (Tabela 2). Além disso, por se tratar de uma pastagem em estágio avançado de degradação, a sua superfície tem um alto grau de compactação, devido à presença de crostas que fazem com que as partículas do solo fiquem mais agregadas, dificultando seu desprendimento e carreamento por escoamento superficial.

Como esperado, a taxa de escoamento (Te) apresentou um rápido aumento na fase inicial de escoamento, estabilizando-se após o pico de escoamento (Figura 1). A CR foi a que mais demorou a apresentar o seu pico de escoamento, estabilizando-se apenas aos 45 minutos. Apesar da $\mathrm{CP}$ e CR terem apresentado as menores perdas de solo, elas geraram Te bem diferentes, uma vez que a $\mathrm{CR}$ tem Te $50 \%$ maior que a CP. O tipo de cobertura vegetal presente pode está influenciando para que CR tenha uma elevada Te, porém uma baixa perda de solo.

Tabela 5. Características da taxa de erosão e do escoamento superficial nas áreas pastagem degradada (PD), terra arada (TA), caatinga regenerada (CR) e caatinga preservada (CP)

\begin{tabular}{ccccc}
\hline Áreas & Ti (minutos) & $\mathrm{C}($ adim. $)$ & $\mathrm{D}_{\mathrm{en}}\left(\mathrm{kg} \mathrm{m}^{-2} \mathrm{~s}^{-1}\right)$ & $\mathrm{P}_{\mathrm{sn}}\left(\mathrm{kg} \mathrm{ha}^{-1}\right)$ \\
\hline $\mathrm{PD}$ & $3,55 \mathrm{~b}$ & $0,72 \mathrm{a}$ & $5,22 \times 10^{-6} \mathrm{~b}$ & $18,87 \mathrm{~b}$ \\
$\mathrm{TA}$ & $5,74 \mathrm{ab}$ & $0,67 \mathrm{ab}$ & $3,28 \times 10^{-5} \mathrm{a}$ & $144,69 \mathrm{a}$ \\
$\mathrm{CR}$ & $6,13 \mathrm{ab}$ & $0,74 \mathrm{~b}$ & $3,52 \times 10^{-6} \mathrm{c}$ & $7,37 \mathrm{c}$ \\
$\mathrm{CP}$ & $8,94 \mathrm{a}$ & $0,40 \mathrm{~b}$ & $1,97 \times 10^{-6} \mathrm{c}$ & $7,26 \mathrm{c}$ \\
\hline
\end{tabular}

Médias seguidas pela mesma letra na linha não diferem entre si pelo teste de Tukey a 5\%. Tempo de início do escoamento (Ti), coeficiente de escoamento superficial (c), porcentagem de precipitação perdida pelo escoamento superficial (PPPSE), taxa de desagregação normatizada (Den) e perda de solo normalizada (Psn). 


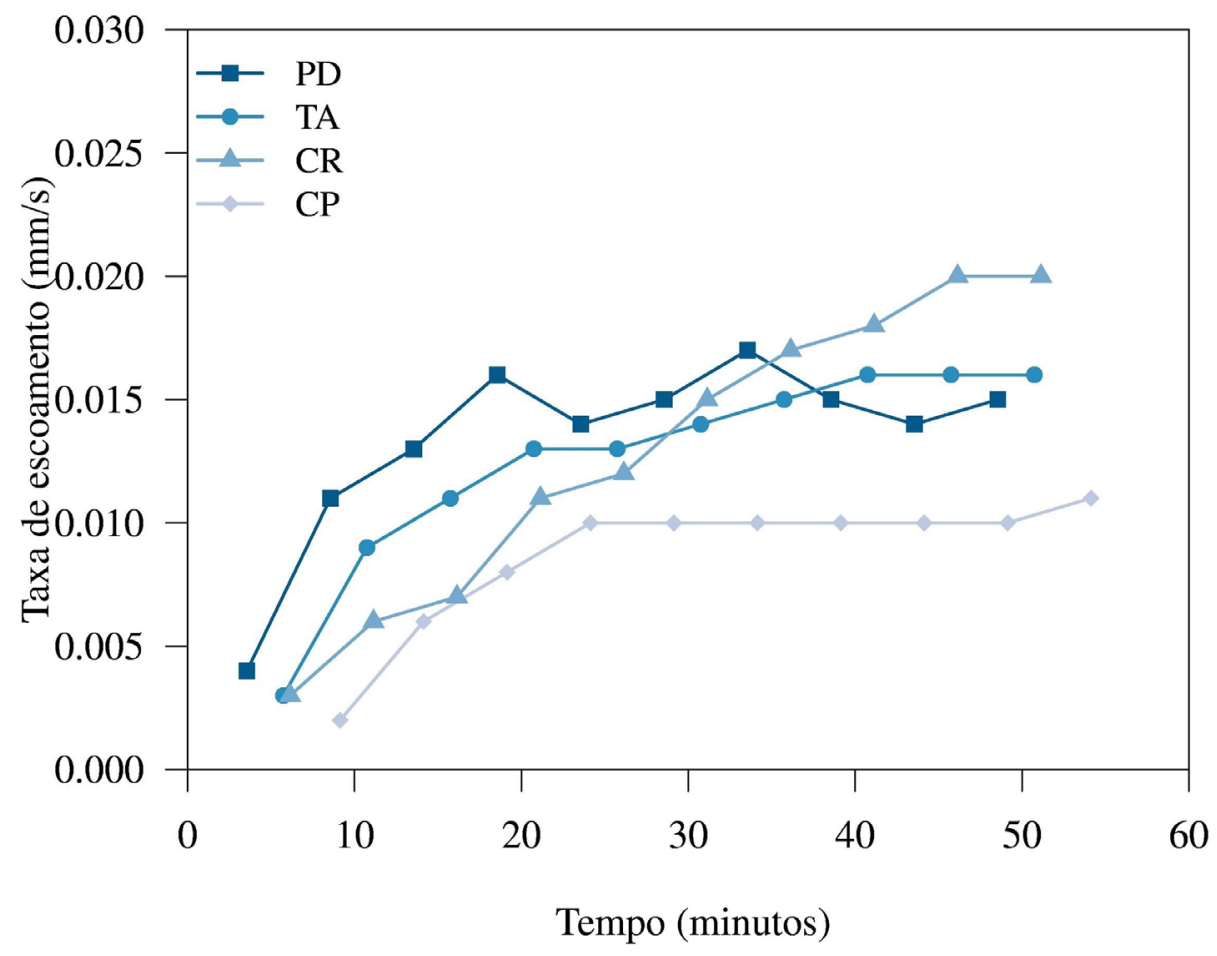

Figura 1. Dinâmica da taxa de escoamento superficial em área de pastagem degradada (PD), terra arada (TA), caatinga regenerada (CR) e caatinga preservada (CP)

Para se determinar a eficiência na redução do escoamento (ERE) e a eficiência na redução dos sedimentos (ERS), consideraram-se como referência para os cálculos as áreas de PD e TA, visto que as mesmas apresentaram as maiores perdas de água e solo, respectivamente. Conforme ilustrado na Figura 2, independente da área, houve uma maior ERS em comparação à ERE. Para CP houve eficiência na redução do escoamento de $35 \%$, para a mesma área observou-se eficiência na redução de sedimentos de $96 \%$. Comportamento semelhante foi encontrado por Wang et al. (2017), que estudaram a eficiência de diferentes práticas agrícolas na redução do escoamento e da erosão e observaram maiores reduções na erosão em confrontação com o escoamento. Percebe-se que a CR obteve valores discrepantes entre ERE e ERS, respectivamente $1,45 \%$ e $96 \%$. A maior eficiência para redução de sedimentos pode está relacionada à cobertura do solo presente na $\mathrm{CR}$, pois, além das plantas herbáceas, a mesma conta com a serrapilheira, que, por conter principalmente folhas e galhos, formam uma barreira de proteção contra o impacto da gota de chuva e carreamento dos sedimentos. A TA, além de apresentar a maior perda de solo, registrou uma ERE de apenas 16\%, ressaltando-se que a utilização da mesma deve está atrelada à prática de conservação do solo e água.

A produção de sedimentos em função do escoamento superficial pode ser visualizada na Figura 3. Nota-se que, apesar de a CR ter um escoamento $15 \mathrm{~mm}$ superior à $\mathrm{CP}$, a diferença de perda de solo entre as áreas é de apenas $0,10 \mathrm{~kg}$. Isso demonstra que a $\mathrm{CR}$ não obteve características que aproximam a perda de água da $\mathrm{CP}$, no entanto conseguiu características que favorecem a diminuição da produção de sedimentos. Ribeiro Filho et al. (2017), estudando o escoamento e perda de solo em caatinga regenerada durante cinco anos, observaram que a mesma alcançou 23 eventos de escoamento e erosão, contra 18 de um caatinga manejada. A TA e a PD desempenharam o mesmo comportamento, aumentado a perda de solo em função da maior lâmina de escoamento, sendo a produção de sedimentos da TA muito superior às demais. A CP, a CR e a PD de até $15 \mathrm{~mm}$ de lâmina escoada apresentam perdas de solo bem próximas, sendo que a partir dessa lâmina as características de solo e cobertura vegetal tendem a influenciar de forma mais significativa na perda de solo. 


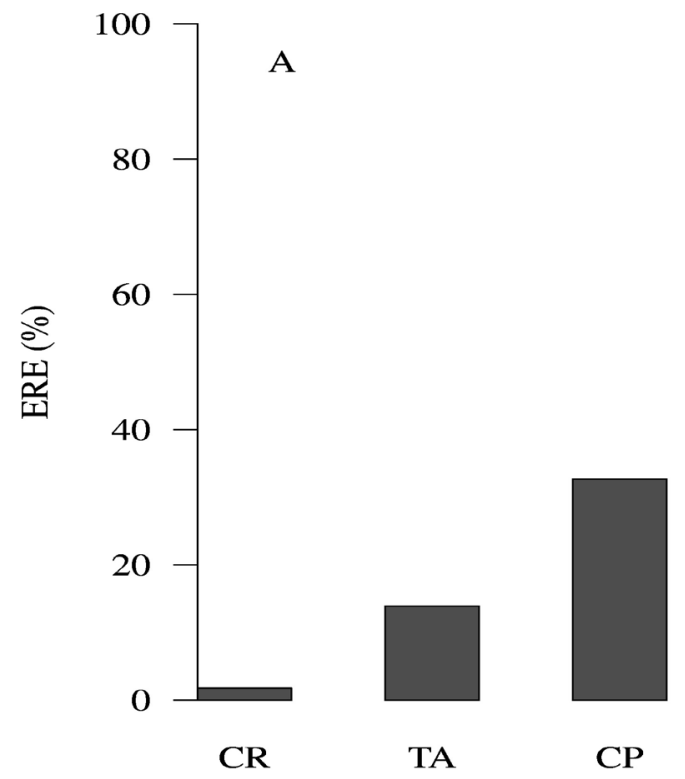

Áreas

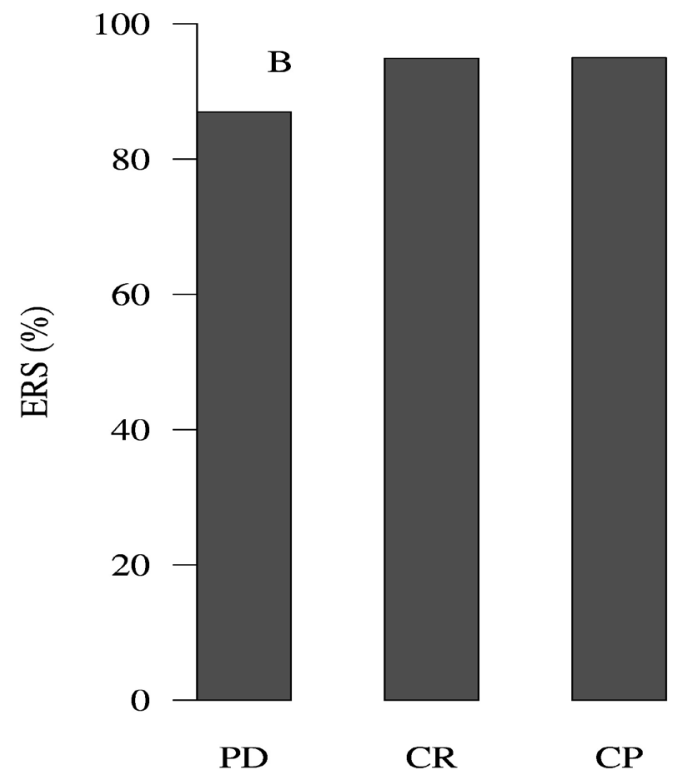

Áreas

Figura 2. Eficiência na redução do escoamento (ERE) (A) e eficiência na redução de sedimentos (ERS) (B) em áreas de pastagem degradada (PD), terra arada (TA), caatinga regenerada (CR) e caatinga preservada $(\mathrm{CP})$

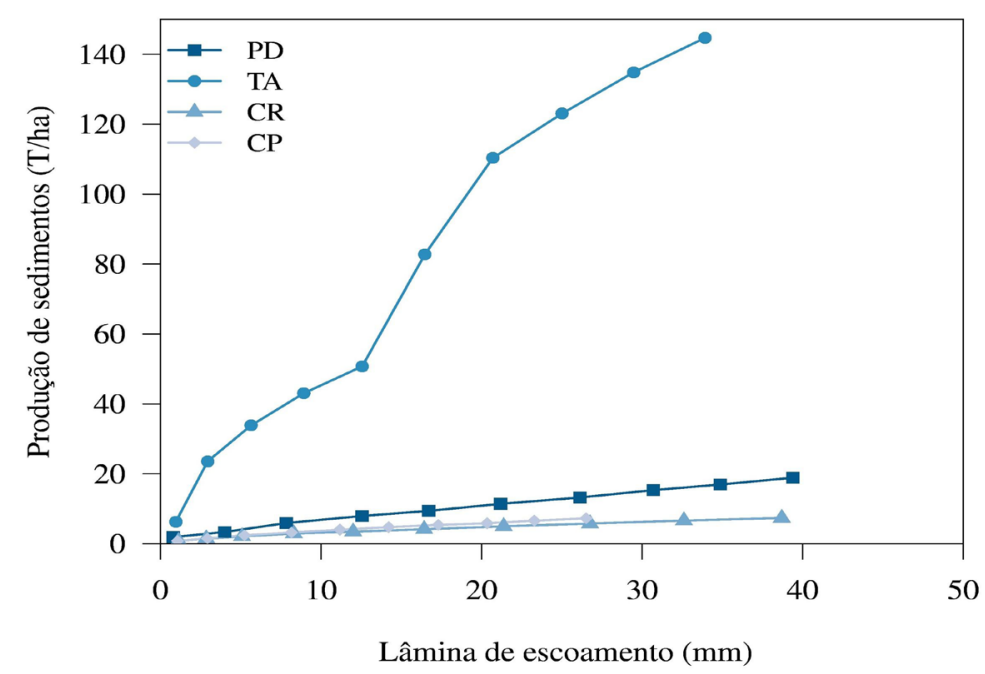

Figura 3. Relação entre a produção de sedimentos e lâmina de escoamento para as áreas de pastagem degradada (PD), terra arada (TA), caatinga regenerada (CR) e caatinga preservada (CP)

\section{CONCLUSÕES}

- Os diferentes usos de solo exerceram efeitos significativos sob a infiltração, o escoamento superficial e a perda de solo.

- A caatinga preservada apresentou a maior taxa de infiltração de água no solo e a menor perda de água e solo.
- A aração não se mostrou uma prática eficiente de manejo do solo, pois a mesma não ocasionou reduções significativas do escoamento superficial, além de aumentar em 7,66 vezes a perda de solo em comparação à pastagem degradada.

- A caatinga regenerada, apesar de sua baixa capacidade de infiltração e alto escoamento, 
adquiriu características que minimizam a perda de solo.

- Por fim, conclui-se que são necessários mais de 30 anos de regeneração natural para que uma área de pastagem adquira características de infiltração próximas a uma área de caatinga preservada.

\section{AGRADECIMENTOS}

Ao Conselho Nacional de Desenvolvimento Científico e Tecnológico (Processo n ${ }^{\circ} 441305 / 2017$ 2); à Fundação de Amparo à Ciência e Tecnologia do Estado de Pernambuco - FACEPE (Processos $\mathrm{n}^{\text {os }}$ APQ-1196-5.03/15 e APQ-0296-5.01/17); e ao Observatório Nacional da Dinâmica da Água e de Carbono no Bioma Caatinga (Edital INCT -MCTI/CNPq/CAPES/FAPs n ${ }^{\circ} 16 / 2014$, Processo $\left.n^{\circ} 465764 / 2014-2\right)$.

\section{REFERÊNCIAS BIBLIOGRÁFICAS}

ABRANTES, J.R.C.B.; LIMA, J.L.P.; MONTENEGRO, A.A.A. Desempenho da modelagem cinemática do escoamento superficial para chuvas intermitentes em solos com cobertura morta. Revista Brasileira de Engenharia Agrícola e Ambiental, Campina Grande, v.19, n.2, p.166-172, 2015.

AGUIAR, M.I.; MAIA, S.M.F.; OLIVEIRA, T.S.; MENDONÇA, E.S.; ARAÚJO FILHO, J.A. Perdas de solo, água e nutrientes em sistemas agroflorestais no município de Sobral, CE. Revista Ciência Agronômica, Fortaleza, v.37, p.270-278, 2006.

BEZERRA, S.A.; CANTALICE, J.R.B. Erosão entre sulcos em diferentes condições de cobertura do solo sob cultivo da cana-de-açúcar. Revista Brasileira de Ciência do Solo, Viçosa, v.30, n.3, p.565-573, 2006.

CASSOL,E.A.; LIMA, V.S. Erosão em entressulcos sob diferentes tipos de preparo e manejo do solo. Pesquisa Agropecuária Brasileira, Brasília, v.38, p.117-124, 2003.
CASSOL, E.A.; CANTALICE, J.R.B.; REICHERT, J.M.; MONDARDO, A. Escoamento superficial e desagregação do solo em entressulcos em solo franco-argiloarenoso com resíduos vegetais. Pesquisa Agropecuária Brasileira, Brasília, Brasília,v.39, p.685-690, 2004.

CANTALICE, J.R.B.; SILVA, M.D.R.; RODRIGUES, J.J.V.; RODAL, M.J.; PESSOA, L.M. Estrutura lenhosa e hidrodinâmica do escoamento superficial em uma área de caatinga. Revista Caatinga, Mossoró, v.12, n.4, p.201-211, 2008.

CANTALICE, J.R.B.; SILVEIRA, F.P.M. ; SINGH, V.P. ; SILVA, Y.J.A.B. ; CAVALCANTE, D.M; SANTOS, C. G. Interrill erosion and roughness parameters of vegetation in rangelands. Catena, Cremlingen, v. 16, p.1-7, 2016.

FRANCO, A.M.P.; CASSOL, E.A.; PAULETTO, E.A.; INDA, A.V. Erodibilidade do solo em entressulcos determinada experimentalmente e por modelos matemáticos em um argissolo vermelho. Revista Brasileira de Agrociência, Pelotas, v.18 n. 2-4, p.175-187, 2012.

FREITAS, F.J.; CANTALICE, J.R.B.; BEZERRA, S.A.; SILVA, M.D.R.O.; SANTOS, P.M.; CORREA, R.M.; LIMA, P.A.; FIGUEIRA, S.B. Erosão em entressulcos sob Caatinga e cultura agrícolas. Revista Brasileira de Ciência do Solo, Viçosa, v.32, p.1743-1751, 2008.

JULIEN, P.Y. Erosion and sedimentation. Melbourne, Cambridge University Press, 1995. 280p.

LEITE, P.A.M.; SOUZA, E.S.; SANTOS, E.S.; GOMES, R.J.; CANTALICE, J.R.; WILCOX, B.P. The influence of forest regrowth on soil hydraulic properties and erosion in a semiarid region of Brazil. Ecohydrology, Western, Edição especial, p.1-12, 2017.

LIMA, C.A.; PALÁCIO, H.A.Q.; ANDRADE, E.M.; SANTOS, J.C.N.; BRASIL, P.P. Characteristics of rainll and erosion under natural 
conditions of land use in semiarid regions. Revista Brasileira de Engenharia Agrícola e Ambiental, Campina Grande, v.17, n.11, p.1222-1229, 2013.

LIMA, C.A.; MONTENEGRO, A.A.A.; SANTOS, T.E.M.; ANDRADE, E.M.; MONTEIRO, A.L.N. Práticas agrícolas no cultivo da mandioca e suas relações com o escoamento superficial, perdas de solo e água. Revista Ciência Agronômica, Fortaleza, v.46, n.4, p.697-706, 2015.

MARIOTI, J.; BERTOL, I.; RAMOS, J.C.; SOUZA WERNER, R.; PADILHA, J.; HENRIQUE BANDEIRA, D. Erosão hídrica em semeadura direta de milho e soja nas direções da pendente e em contorno ao declive, comparada ao solo sem cultivo e descoberto. Revista Brasileira de Ciência do Solo, Viçosa, v.37, n.5, 2013.

PANACHUKI E.; BERTOL, I.; ALVES SOBRINHO, T.;OLIVEIRA,P.T.S.;RODRIGUES, D.B.B. Perdas de solo e de água e infiltração de água em latossolo vermelho sob sistemas de manejo. Revista Brasileira de Ciências do Solo, Viçosa, v.35, n.1, p.1777-1785, 2011.

PARENTE, H.N.; SILVA, D.S.; ANDRADE, A.P.; SOUZA, E.S.; ARAÚJO, K.D.; MAIA, M.O. Impacto do pisoteio caprino sobre atributos do solo em área de caatinga. Revista Brasileira de Saúde e Produção Animal, Salvador, v.11, n.2, p.331-34, 2010.

RASBAND, W.S. ImageJ. U.S. National Institute of Health, Bethesda, Maryland, USA 2012.

RIBEIRO FILHO, J.C.; PALÁCIO, H.A.Q.; ANDRADE, E.M.; SANTOS, J.C.; BRASIL, J.B. Raifall characterization and sedimentological responses of watersheds with diferemt land uses to precipitation in the semiarid region brazil. Revista Caatinga, Mossoró, v.30, n.2, p.468-478, 2017.

RODRIGUES, V.A.; SÁNCHEZ-ROMÁN, R.M.; TARJUELO, J.M.; SARTORI, M.M.P.; CANALES, A.R. Avaliação do escoamento e interceptação da água das chuvas. Revista Irriga, Botucatu, v.especial, p.1-13, 2015.
SILVA, P.F.; LIMA, J.R.S.; ANTONINO, A.C.D.; SOUZA, R.; SOUZA, E.S.; SILVA, J.R.I.; EDEVALDO, M.A. Seasonal patterns of carbon dioxide, water and energy fluxes over the Caatinga and grassland in the semi-arid region of Brazil. Journal of Arid Environments, v.147, p.71-82, 2017 a.

SILVA, J.R.I.; SOUZA, R.M.S.; SANTOS, W.A. ALMEIDA, A.Q.; SOUZA, E.S.; ANTONINO, A.C.D. Aplicação do método de Budyko para modelagem do balanço hídrico no semiárido brasileiro. Scientia Plena, Aracajú, v.13, n.10, $2017 \mathrm{~b}$.

SANTOS, W.M.; SOUZA, R.M.S.S.; SOUZA, E.S.; ALMEIDA, A.Q.; ANTONINO, A.C.D. Variabilidade espacial da sazonalidade da chuva no semiárido brasileiro. Journal of Environmental Analysis and Progress, Recife, v.2, n.4, p.368376, 2017.

SANTOS T.E.M.; MONTENEGRO, A.A.A.; SILVA JUNIOR, V.P.; Erosão hídrica e perda de carbono orgânico em diferentes tipos de cobertura do solo no semiárido, em condições de chuva simulada. Revista Brasileira de Recursos hídricos, Porto Alegre, v.13, n.2, p.113-125, 2008.

SINGH, V.P. Analytical solutions of cinematic for erosion on a plane: II Rainfall of finite duration. Advances in Water Resouces, v.6, p.88-95, 1983.

SIMONS, D.B. \& SENTURK, F. Sediment transport technology: Water and sediment dynamics. Fot Collins, Water Resources Publications. 1992. 897p.

SOUZA, R.M.S.; SOUZA E. S.; ANTONINO, A.C.D.; LIMA J.R.S. Balanço hídrico em área de pastagem no semiárido pernambucano. Revista Brasileira de Engenharia Agrícola e Ambiental, Campina Grande, v.19, n.5, p.449-455, 2015.

ZHAO, X.; CHEN, X.; HUANG, J.; WU, P.; HELMERS, M.J. Effects of vegetation cover of natural grassland on runoff and sediment yield in 
EFEITO DE DIFERENTES USOS DO SOLO NA EROSÃO HÍDRICA EM REGIÃO SEMIÁRIDA

loess hilly region of China. Journal of the Science of Food and Agriculture, v.94, n.3, p.497-503, 2014.

WANG, L.; DALABAY, N.; LU, PEI.; WU, F. Effects of tillage practices and slope on runoff and erosion of soil from the Loess Plateau, China, subjected to simulated rainfall. Soil \& Tillage Research, v.166, n.1, p.147-156, 2017.

WOOLHISER. D.A.; LIGGETT, J.A. Unsteady onedimensional flow over a plane - The rising hydrograph. Water Resources Research, Willey, v.3, n.3, p.753-771, 1967. 